This item was submitted to Loughborough's Research Repository by the author.

Items in Figshare are protected by copyright, with all rights reserved, unless otherwise indicated.

\title{
Formulating polyurethanes using case based reasoning
}

PLEASE CITE THE PUBLISHED VERSION

PUBLISHER

(c) Maney

VERSION

VoR (Version of Record)

LICENCE

CC BY-NC-ND 4.0

REPOSITORY RECORD

Segura-Velandia, Diana M., Richard J. Heath, and Andrew A. West. 2009. "Formulating Polyurethanes Using Case Based Reasoning". figshare. https://hdl.handle.net/2134/4231. 
This item was submitted to Loughborough's Institutional Repository (https://dspace.lboro.ac.uk/) by the author and is made available under the following Creative Commons Licence conditions.

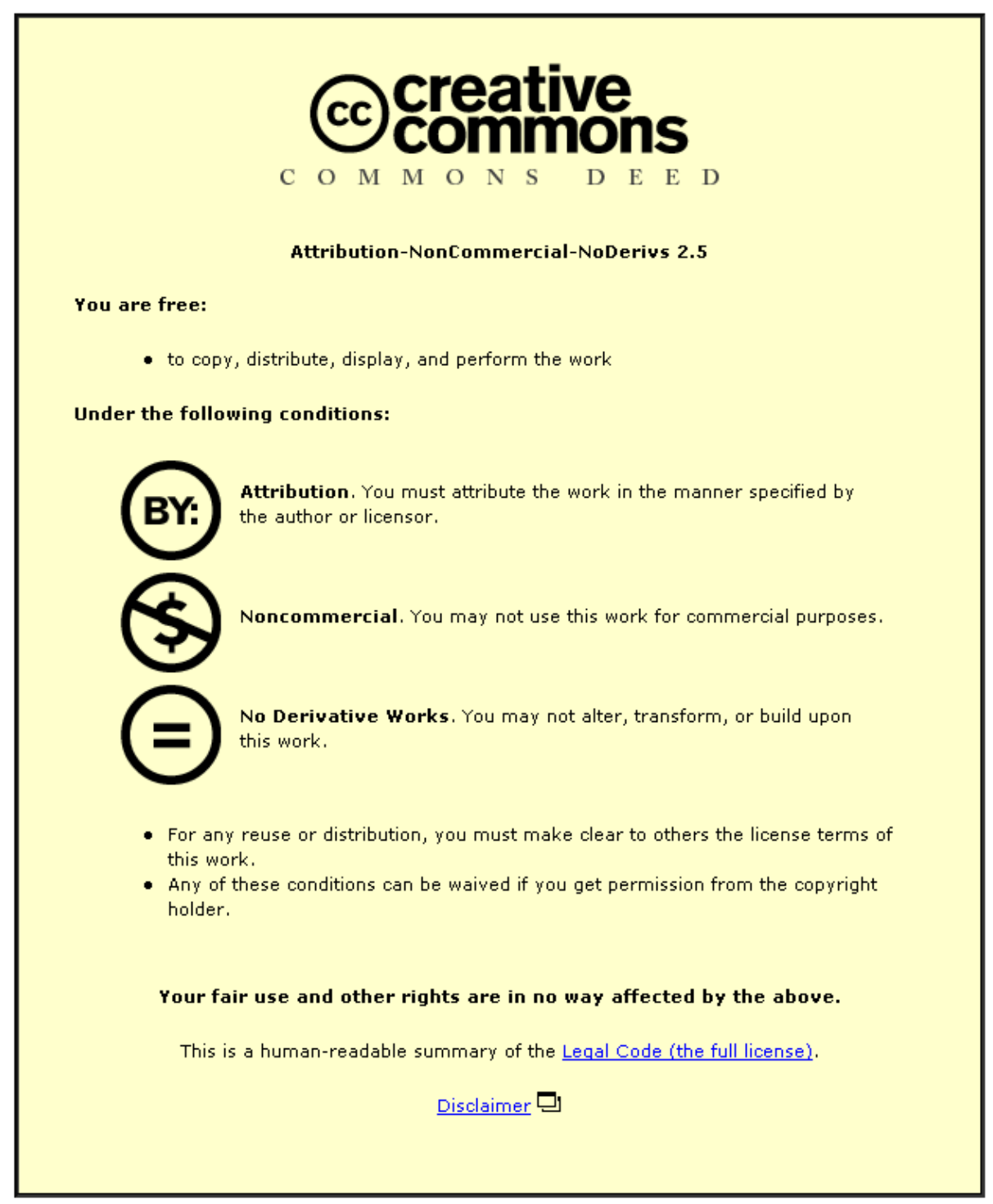

For the full text of this licence, please go to: http://creativecommons.org/licenses/by-nc-nd/2.5/ 


\title{
Formulating polyurethanes using case based reasoning
}

\author{
D. M. Segura Velandia*, R. J. Heath and A. A. West
}

A large amount of historical knowledge exists in the form of 'formulation experiences' across polyurethane manufacturing companies. This knowledge is difficult to formalise, share and use in new formulations. As a part of an effort to support the polyurethane formulating problem, the use of case based reasoning (CBR) has been assessed. Two basic problems in the development of the proposed hybrid tool that uses past formulations to solve new problems are studied. The problems investigated are related to the retrieval of former formulations that are similar to a new problem description by the CBR module, and the adaptation of the retrieved case to meet the problem constraints using an artificial neural network (ANN). Results indicated that the CBR-ANN system is useful for reusing historical data. Although the obtained ANN is unable to generalise well when presented with more data independent from the original data set, results proved that real formulation data can be used as a 'knowledge repository' that can guide CBR adaptation without human expert intervention.

Keywords: Case based reasoning, Polyurethane formulation, Artificial neural network

\section{Introduction}

Polyurethane (PU) formulations have been developed mostly by trial and error methods which have resulted in many innovative and useful materials and a large amount of historical formulation data. Owing to their versatile chemistry, PUs can be tailor made into foams, adhesives, sealants and elastomers for many industrial and everyday applications (e.g. automotive, ${ }^{1,2}$ construction, ${ }^{3}$ electronics, ${ }^{4}$ environmental and medical ${ }^{5,6}$ ). Paradoxically, this versatility makes the reuse of PU formulations difficult for non-experts. This is due to complex interactions between formulation components further affected by processing conditions. The formulation of PUs often involves reasoning about several abstract concepts such as microstructure, interpretations and heuristic 'rules of thumb' based on experience. The four interacting types of knowledge required when developing new PU formulations (Fig. 1) include: the composition, structure, manufacturing or processing conditions, and final product properties.

Several researchers have attempted to relate changes in composition and manufacturing variables to the microand macro-structure and to final properties of PUs. ${ }^{7-9}$ These structure-property relationship studies aim for the development of mathematical models that can support formulators when developing new formulations.

Although these studies have provided with crucial knowledge on how formulation and processing

Wolfson School of Mechanical and Manufacturing Engineering Loughborough University, Leics, LE11 3TU, UK

*Corresponding author, email d.segura@lboro.ac.uk parameters affect PU properties for various applications, their ability to predict accurately and effectively PU properties has not been achieved.

Statistical designs have also been used to assist the development of new PU formulations. Most statistical designs require both the identification of the variables that greatly influence a PU system and the identification of the levels at which those variables will lead to stable polyurethane products. These decisions rely greatly on an expert's experience and on the type of design to be carried out. For example, in situations where a product is to be introduced, screening designs are best suited. ${ }^{10,11}$ Subsequent optimisation and tuning of the statistical design is also knowledge intensive and hence expert dependent. An advantage of experimental designs compared to formulating by first principles is that they allow the formulator to understand, by constructing predictive models, the effect of the variables on the PU properties. Even though the designs might not result in any commercially viable PU formulations, statistical analysis of unsuccessful products can still shed some light on the understanding of the PU formulations triad effect (e.g. composition, structure, manufacturing) on the resultant product properties.

The degree of success of developing new formulations or modifying existing ones to achieve desired end product properties depends on the choice of the raw materials, the processing conditions, and to a large extent on existing knowledge (i.e. previous formulations, and the skills and expertise or experiences of the formulator). The traditional approach to reuse that expertise often lacks a consistent and systematic procedure. As a consequence, formulations for a variety of applications generated in an ad hoc manner lead to a 


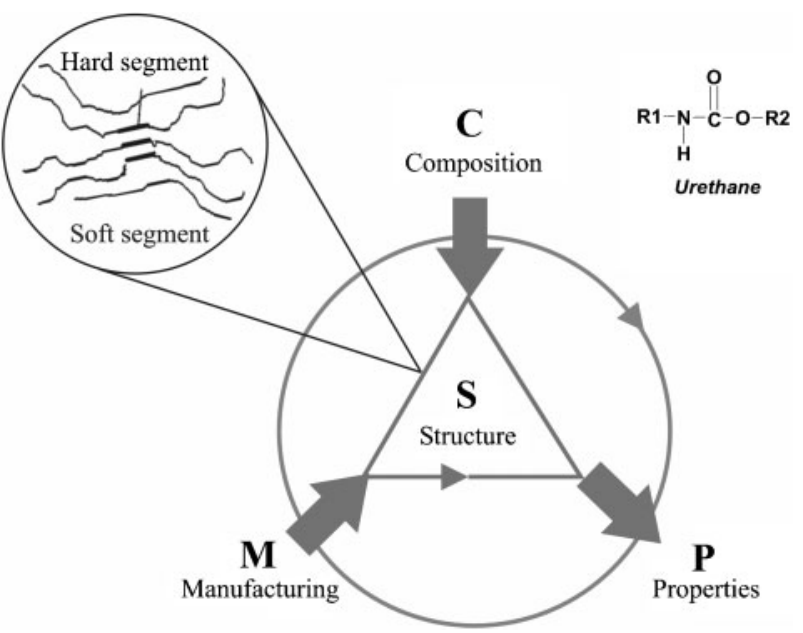

1 Interrelation between PU's layers: composition structure and manufacturing process that influence design of new formulations

lack of understanding of the mechanisms that could explain how and why the formulations work. Clearly, novel approaches that enable effective PU formulation by reusing past experiences and expertise while at the same time provide a framework for collecting and sharing this formulation knowledge for future problem solving are required.

\section{Case based reasoning (CBR)}

In the recent years, the ideas and techniques that have been developed by the Artificial Intelligence (AI) community have been applied in complex domains such as engineering, business management, medicine, and design and wherever an organisation wants to enhance its ability and capacity to perform, compete, and cope with change. ${ }^{12}$ Lessons learnt in AI in knowledge acquisition, representation, inference, search, and learning can be brought about for assisting formulators to develop new formulations by reusing formulation experiences.

For successful knowledge reuse and management, the application of the CBR methodology has been studied in various formulated product applications. Recent applications include aluminium alloys, ${ }^{13,14}$ steel manufacturing, ${ }^{15,16}$ formulation of pharmaceuticals, ${ }^{17,18}$ rubbers formulation ${ }^{19}$ and plastics manufacturing. ${ }^{20}$ Similarly to the PU domain, the drivers for developing these knowledge based systems were concerned mainly with:

(i) the practice of the discipline being regarded more as an art rather than as a science and therefore the domain was poorly understood

(ii) no comprehensive mathematical models to support product design and manufacture exists

(iii) there is a need to formalise such experiential knowledge for satisfactory future problem solving

(iv) there is a need for collecting, structuring, and representing manufacturing knowledge from human experts using a computational system to solve problems and support learning through time.

In CBR, the model of human reasoning is one where human experts solve new problems by adapting solutions that were used to solve old problems (Fig. 2). ${ }^{21,22}$ An expert system's domain knowledge built under this

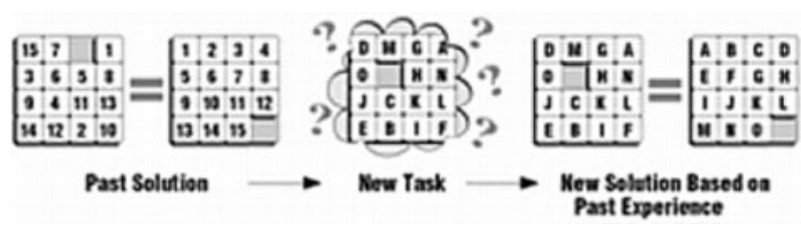

2 Case based reasoning expertise comprises experience; when solving new problem, experts rely on past episodes; case based reasoning is general paradigm for reasoning from experience

model would be composed of a set of 'experiences' that can be retrieved and adapted to solve new problems. This paradigm of problem solving is referred to as CBR.

In order to understand why CBR is suited for the PU formulation domain, a description of the PU formulators' reasoning is presented next.

\section{Formulating PUs using CBR methodology Polyurethane formulators reasoning}

The tasks that describe the reasoning process to find a formulation that meets given constraints is described as follows (see Fig. 3):

(i) problem description: in particular, the problem of formulating a PU is usually described by a set of constraints, which in turn are generally expressed in terms of mechanical properties of the material e.g. 'tensile strength must be higher than $\mathrm{x}$, and hardness must be lower than $\mathrm{y}^{\prime}$

(ii) selection of known/similar properties to design target: once property constraints are specified and the problem is fully described, formulators draw upon different types of knowledge to select some baseline formulation to begin their search for a solution. They usually start with a similar formulation to the design target

(iii) alteration of properties by changes in composition and processing: the formulation process involves an iterative approach to change the characteristics of the materials hopefully in the direction of the target properties. Formulators look for changes that can be made to the materials or processing conditions in order to improve the properties. However, this complex task is only efficiently done by experts in the $\operatorname{area}^{23}$

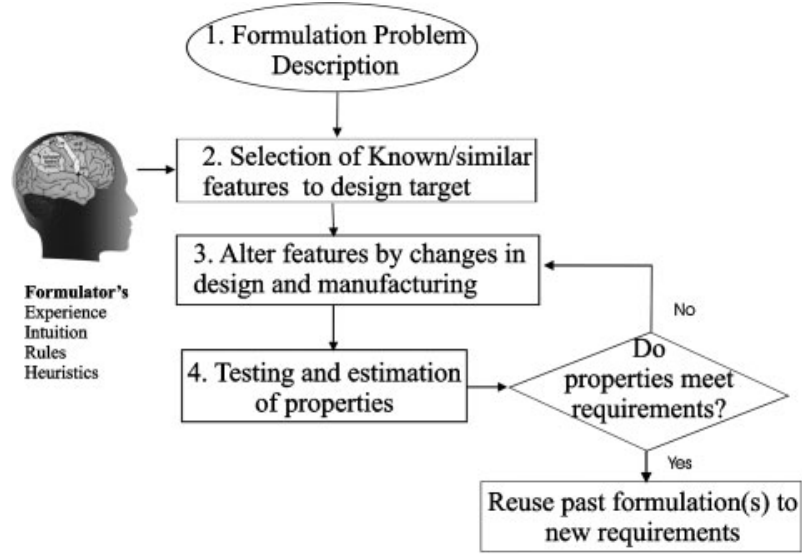

3 Polyurethane formulation process formulator's reasoning cycle 


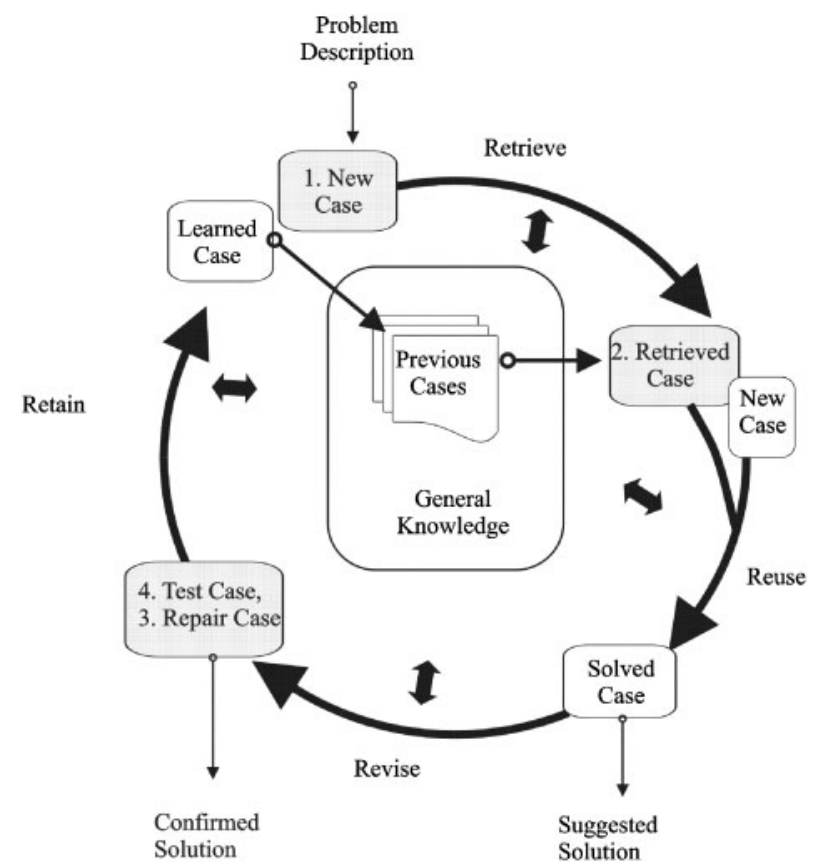

4 Basic CBR flow

(iv) testing and estimation of properties: after a group of possible formulations are identified likely to meet the specifications, tests and analysis of the data give rules that are more accurate and provide the basis for a finer set of experiments in the next iteration.

\section{Reasoning through cases}

Conceptually, CBR is commonly described as a cyclic process (see Fig. 4) ${ }^{24}$ First, a problem situation or query is described. Then, a similarity measure is computed so that the most similar cases to the query are retrieved. The information in the retrieved case is then reused to provide an initial solution to the problem. If this does not fully satisfy the problem specifications, the retrieved case's solution is adapted. Once the retrieved case has been adapted, this candidate solution is tested. If the test is not successful, sources of failure are investigated and the partial solution is repaired and tested again until eventually a suitable solution is found. Comparing Fig. 4 with Fig. 3, it can be seen that PU formulators reason in the way $\mathrm{CBR}$ solves problems. Therefore, CBR would be a suitable methodology to enable the effective use of historical formulation knowledge while providing a computational framework to collect and share this experiential knowledge. In addition, due to the fact that the formulation of PUs is an iterative process that can be both time consuming and expensive, a
Table 2 Foam expert's selected variables levels to yield stable foam

\begin{tabular}{|c|c|c|c|c|c|}
\hline \multirow{2}{*}{$\begin{array}{l}\text { Factors } \\
\text { Polyether polyol } 1\end{array}$} & \multicolumn{5}{|c|}{ Levels, pbw } \\
\hline & \multicolumn{5}{|c|}{ Vary to keep formulation at $100 \%$} \\
\hline Polyether polyol 2 & 1.5 & 2 & $2 \cdot 5$ & 3 & \\
\hline Amine cross-linker & 0 & 0.5 & 1 & 1.5 & 2 \\
\hline Amine catalyst 1 & $0 \cdot 3$ & 0.5 & $0 \cdot 7$ & $0 \cdot 9$ & \\
\hline Amine catalyst 2 & 0.25 & 0.5 & $0 \cdot 75$ & & \\
\hline Surfactant & $0 \cdot 25$ & 0.5 & $0 \cdot 75$ & & \\
\hline Water & 3 & $3 \cdot 25$ & 3.5 & $3 \cdot 75$ & \\
\hline Index & 85 & 100 & & & \\
\hline
\end{tabular}

computational automated approach is expected to generate formulations faster (reduced lead times), and at lower development costs, with greater ease of processing and design flexibility if 'what-if' simulation scenarios are enabled.

The following sections of the present paper address the following:

(i) the codification of available experimental formulation data within the CBR framework (explained in section 'Polyurethane formulations')

(ii) the retrieval of former PU cases similar to a new problem description (shown in section 'Case representation')

(iii) the strategy used to adapt retrieved cases by using neural networks that can generate a mapping between change in mechanical properties and formulation ingredients (covered in section 'Case retrieval')

\section{Methodology and experimental setup}

\section{Polyurethane formulations}

The foam formulation used was provided by Elastogran, BASF-UK (see Table 1). It corresponds to a typical combustion modified high resilient foam formulation, which finds use in various automotive foam parts. Following a fractional factorial $\operatorname{design}^{25}$ a set of formulations were obtained. The factors (in total eight) were varied between their maximum and minimum levels according to Table 2.

The foams were produced using conventional PU hand mixing methods. Five response variables were recorded. These included the bulk density not the true polymer density, ${ }^{26}$ tensile strength, elongation percentage, compression set, and hardness. After $24 \mathrm{~h}$ of conditioning at room temperature, test of the PU foam samples were performed. Hardness was measured according to the standard test BS ISO 2439:2000. ${ }^{27}$ Tensile strength (the maximum tensile stress applied during stretching to rupture) and elongation at break

Table 1 Combustion modified high resilient foam formulation

\begin{tabular}{llllc}
\hline Component & OH value & Mol. weight & Functionality & Formulation, ppw \\
\hline Polyether Polyol 1 & 28 & 5400 & $2 \cdot 7$ & $91 \cdot 25$ \\
Polyether Polyol 2 & 42 & 150 & 3 & $2 \cdot 5$ \\
Amine Cross-linker & 1160 & - & 3 & 1 \\
Amine Catalyst 1 & - & - & - & $0 \cdot 7$ \\
Amine Catalyst 2 & - & - & 2 & $0 \cdot 5$ \\
Surfactant & - & 18 & & 3.55 \\
water & - & & & \\
\%NCO & $33 \cdot 1$ & & & \\
index & $85-105$ & &
\end{tabular}




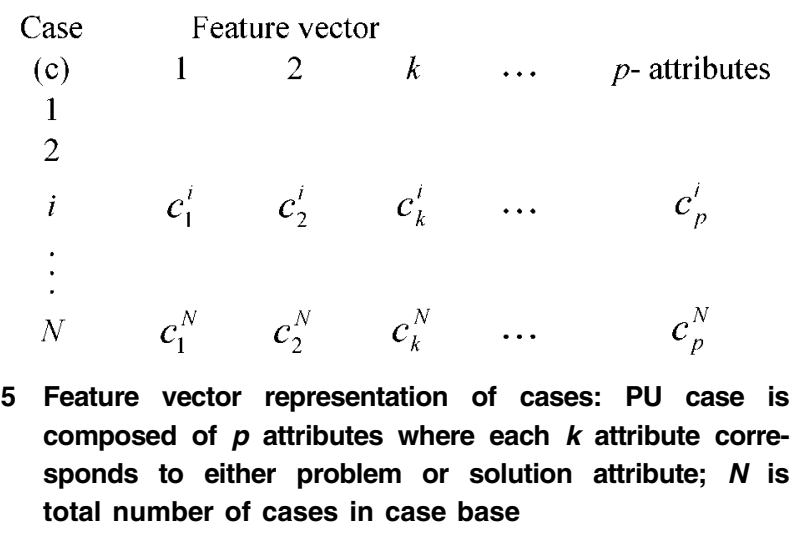

(the percentage of elongation at rupture) were measured according to BS EN ISO 1798:2000. ${ }^{28}$ Compression set was measured according to BS EN ISO $1856: 2001 .^{29}$

\section{Case representation}

In CBR a 'case' represents a previous experience or problem $^{30}$ and it is composed of a 'problem description', a 'problem solution', and an 'outcome', which is a measure of success of the applied solution in real conditions. In particular, a 'PU case' can be described by a set of attributes that correspond to PU formulation specifications (e.g. materials, ratios and processing methods) and its associated final properties.

Therefore, for the implementation of CBR system, the PU formulations or recipes were coded into 'cases'. Data regarding both the chemical formulations to produce PU foams, and testing data showing the performance of those of PU foams were used to represent the case solution and the case problem respectively. Case problem's attributes consist of the five physical properties measured for the PUs. The case solution's attributes correspond to eight attributes, which are the chemical formulation variables.

These PU cases have an attribute value representation format (see Fig. 5), which is the preferred format because formulations are normally presented as a list of ingredients and processing conditions with their corresponding values. In similar domains, other researchers have used this format for the representation of formulation cases. ${ }^{17,18,31,32}$

For a particular case $i$ the feature case vector $c$ is

$$
\boldsymbol{c}^{(\mathrm{i})}=\left\{c_{1}^{\mathrm{i}}, c_{2}^{\mathrm{i}}, \ldots c_{\mathrm{k}}^{\mathrm{i}}\right\}
$$

The set of all $c^{(\mathrm{i})}$ 's constitutes the case base (CB)

$$
C B=\left\{c^{(\mathrm{i})}, \ldots c^{(\mathrm{N})}\right\}
$$

\section{Case retrieval}

Using each case as a query, the nearest neighbour rule ${ }^{33}$ was implemented using the Euclidean distance

$$
d_{\mathrm{ij}} \equiv\left(\sum_{\mathrm{k}=1}^{\mathrm{p}}\left(x_{\mathrm{ik}}-x_{\mathrm{jk}}\right)^{2}\right)^{1 / 2}
$$

where $x_{\mathrm{ik}}$ denotes the value that the $k_{\mathrm{th}}$ quantitative variable takes for the $i$ th object $(i=1, \ldots, n ; k=1, \ldots, p)$ and $w_{\mathrm{k}}(k=1, \ldots p)$ are non-negative weights associated with the variables.
In particular, the case vector

$$
c^{\prime \mathrm{N}} \in\left\{c^{(\mathrm{i})}, \ldots c^{(\mathrm{N})}\right\}
$$

is said to be the nearest neighbour of $\boldsymbol{q}$ if

$$
\min _{\mathrm{i}} \mathrm{d}\left(\boldsymbol{c}_{\mathrm{i}}, \boldsymbol{q}\right)=\mathrm{d}\left(\boldsymbol{c}^{\prime \mathrm{N}}, \boldsymbol{q}\right)
$$

where $\mathrm{d}\left(\boldsymbol{c}_{\mathrm{i}}, \boldsymbol{q}\right)$ is the distance between the case $\boldsymbol{c}_{\mathrm{i}}$ and the query $q$ calculated for each case in the CB.

Feature weights were set to one, i.e. $\forall_{\mathrm{k}}\left\{w_{\mathrm{k}}=1\right\}$.

\section{Case adaptation}

In CBR, case adaptation is one of the most challenging aspects in the implementation of useful CBR systems and yet it remains the least developed. ${ }^{34}$ Although case adaptation is essential it is a difficult task. It is essential because solutions are never identical to past solutions and frequently two or more previous solutions must be combined to solve a new problem. ${ }^{35}$ Knowledge to adapt cases is difficult to acquire because a deep understanding of the problem domain (at expert level) is required. ${ }^{31,36}$ It is therefore, not surprising that existing commercial CBR tools do not include a well developed adaptation framework.

Recent studies have proposed the use of inductive techniques to ease the adaptation knowledge bottleneck by learning from cases (i.e. historical formulation knowledge). ${ }^{36,37}$ Like inductive learning programs, artificial neural networks (ANN) can be used to learn domain knowledge from examples. The present paper explores the ANN approach as it has received little attention as a tool for CBR adaptation. Specifically, the present work studies how ANNs can induce knowledge from a $\mathrm{CB}$ to reduce the adaptation knowledge acquisition task and how an ANN can support the case adaptation task.

Case adaptation, within the CBR framework, stands for the modification of a retrieved case that partially matches a user query to solve a proposed problem. In particular, for a PU formulation application, case adaptation is expressed in terms of how to modify a retrieved formulation. This encompasses the identification of both the formulation ingredients and their quantities that meet a set of performance constraints.

For the adaptation of retrieved PU cases, the authors studied the feasibility of an ANN learning a desired mapping from experimental PU formulations in order to prove that the $\mathrm{CB}$ has useful implicit knowledge that can be exploited by a neural network and that the knowledge can be used to adapt retrieved cases. A two hidden layer feed forward neural network was trained to map case feature differences to PU formulation properties using the back propagation algorithm (Fig. 6).

\section{Neural network data set}

Differences between problem descriptions (i.e. mechanical properties of a pair of foams) were obtained and used as input patterns and the differences between solution descriptions (i.e. formulation ingredients) as the output patterns. Other researchers ${ }^{37,38}$ have similarly suggested the use of these differences between the case problem features to build adaptation cases.

In order to obtain a large set of input patterns for the training the network, delta property values were calculated using all but the probe cases of the case base, i.e. 29 cases 28 times resulting in 812 differences. Each 


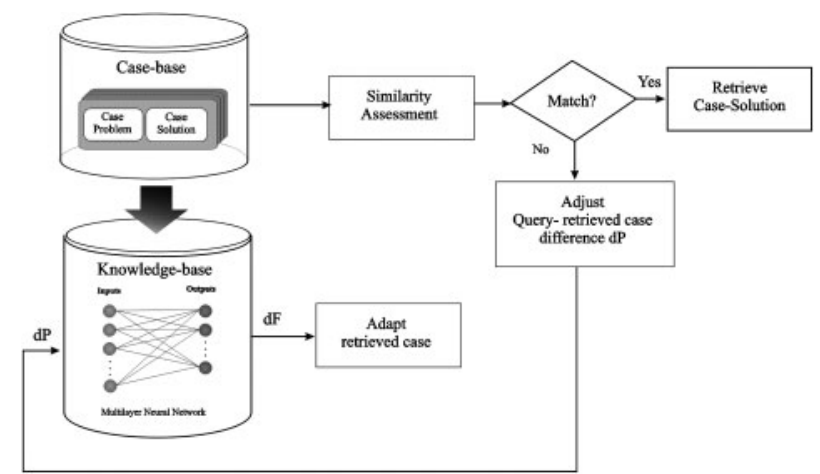

6 Artificial neural network as knowledge base to assist CBR adaptation

input pattern $\delta_{\mathrm{p}}$ is obtained by taking the differences between the case problem attributes for the cases stored in the $\mathrm{CB}$.

Input patterns

$$
\delta p=c^{(\mathrm{i})}-c^{(\mathrm{j})}=\left\{\begin{array}{c}
\text { case } i_{\text {problem }}-\text { case } j_{\text {problem }} \\
\text { attribute k k }
\end{array}\right\},
$$

for case $i \neq j$.

where $c^{(\mathrm{i})}$ is the $i$ th feature vector, which contains only the problem attributes of a case, i.e. the mechanical properties.

In a similar fashion, the outputs of the network are obtained by calculating the differences between the solution attributes of a case; this is between the formulation ingredients.

Targets network

$$
\delta f=c^{(\mathrm{i})}-c^{(\mathrm{j})}=\left\{\begin{array}{c}
\text { case } i_{\text {solution }}-\text { case } j_{\text {attribute k }} \\
\text { attribute }
\end{array}\right\},
$$

for case $i \neq j$.

It is well known that the network's ability to learn any nonlinear function depends heavily on the quality of the data presented to it. ${ }^{39}$ For this reason, once the differences between attribute values were calculated, the input patterns for the network were normalised using the min-max normalisation in the interval $[-1$, 1]. ${ }^{39}$ In addition two types of output data were used, real valued and binary valued i.e. normalised to the interval $[0,1]$.

\section{Network training}

Without enough formulations to justify splitting the data into training and test sets, the cross-validation approach was adopted. ${ }^{39}$ By following this approach the training set was partitioned into five distinct segments.

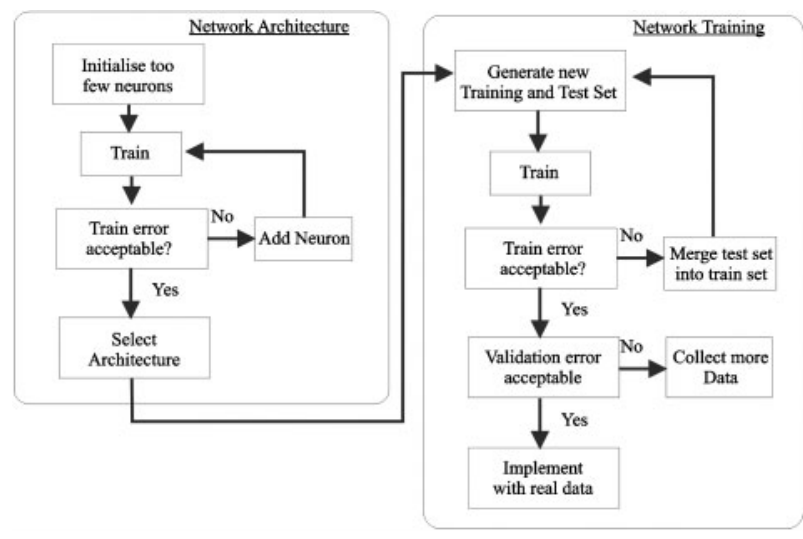

7 Methodology followed to find optimum network architecture and to train network

Each sample in the training set completely specifies all the inputs as well as the outputs that are desired when those inputs are presented. Five networks therefore were trained, each time using random chosen training sets with 792 exemplars and training test sets with 20 exemplars. The network with the lowest error with respect to the training test set was selected. The performance of the selected network was confirmed by measuring its performance on a third independent set of data called a test set. If the error in the test set is not acceptable, a common practice is to joint the training and training test set and retrain. ${ }^{39}$

\section{Network architecture}

The network architecture is the description of the number of the layers in an ANN, each layer's transfer function, the number of neurons per layer, and the connections between layers. Finding the correct network architecture is a not an easy task. Aside from the number of neurons in a network's output layer, the number of neurons in each layer can be found by trial and error. A large body of research exists which suggest several rules of thumb that can be applied to the problem at hand. ${ }^{39-42}$ For the problem studied in the present work a systematic change in the number of number of neurons in each layer was implemented to find an optimum network's architecture (Fig. 7).

The network parameters used are presented in the Table 3.

\section{Results and discussion}

Results using the CBR system using four probe data points are presented as follows. A user query was first presented to the CBR system, and this retrieved the closest formulations to it according to some measure of

\section{Table 3 Network training parameters}

\begin{tabular}{ll}
\hline Function & Name \\
\hline Inputs & 5 real valued variables $[-1,1]$ \\
Outputs & 24 binary valued variables $[1,0]$ \\
Training function & Levenberg-Marquardt backpropagation algorithm \\
Performance function & Mean squared error \\
Transfer function & Sigmoid transfer function \\
Learning function & Gradient descent with momentum weight and bias learning function \\
Maximum number of epochs to train & 100 \\
Performance goal & $1 \mathrm{e}-5$ \\
Minimum performance gradient & $1 \mathrm{e}-10$ \\
\hline
\end{tabular}


similarity, e.g. Euclidean distance (3). Then, the normalised differences between the probe and the case problems were calculated and presented to the trained neural network to obtain the adapted formulation (see results in Table 4).

A two hidden layer feed forward back propagation ANN with binary valued outputs met the goal of the present work, i.e. 'to adjust a retrieved formulation to find the ingredients and their quantities that result in a PU formulation that approximate the user query or requirements'. As the results shown in Table 4, the trained network was able to recognise relationships between case problem feature differences and case solution feature differences using real PU formulation data and it was used successfully to diagnose formulation changes required for adaptation of retrieved cases using a sample of four probe cases. Results for the four probe cases showed that the prediction error (i.e. square error) is low for most of the formulation variables and hence is an indication of good prediction capabilities for the CBR-NN system.

Although the obtained network is unable to generalise well when presented with more data independent from the original data set, this network proved that real formulation data in the $\mathrm{CB}$ can be used as a 'knowledge repository' that can guide CBR adaptation without human expert intervention. The ANN, having memorised a set of data presented to it, outperforms human PU expert capabilities to retrieve a formulation given a set of mechanical properties (i.e. a user query) in the sense that a human expert cannot produce at such detail a PU formulation.

The lack of generalisation could be due to the fact that the network was trained with a very small data set and hence it is not able to discover the relationships between the large numbers of variables involved, which make the problem complex. In addition, the chemistry of PUs is very sensitive to changes in any PU reactive chemical group (e.g. isocyanate, hydroxyl, amine) produced either by changes in processing conditions (e.g. temperature) or by introduction of chemicals intentionally (i.e. increase in formulation ingredients) or unintentionally (i.e. contamination, environmental humidity changes). A

Table 4 Predicted formulation using CBR-NN system Ingredients*

\begin{tabular}{|c|c|c|c|c|c|c|c|c|}
\hline Name & 1 & 2 & 3 & 4 & 5 & 6 & 7 & 8 \\
\hline \multicolumn{9}{|c|}{ Known formulation } \\
\hline probe11 & $90 \cdot 95$ & 3 & $1 \cdot 5$ & 0.3 & $0 \cdot 25$ & $0 \cdot 3$ & $3 \cdot 75$ & 110 \\
\hline probe12 & $90 \cdot 85$ & $1 \cdot 5$ & $1 \cdot 5$ & 0.9 & $0 \cdot 75$ & 0.8 & $3 \cdot 75$ & 85 \\
\hline probe22 & $94 \cdot 2$ & $1 \cdot 5$ & 0 & $0 \cdot 3$ & $0 \cdot 75$ & $0 \cdot 3$ & 3 & 85 \\
\hline probe25 & $90 \cdot 7$ & 3 & $1 \cdot 5$ & 0.3 & 0.75 & 0.8 & 3 & 85 \\
\hline \multicolumn{9}{|c|}{ Adapted retrieved formulation } \\
\hline adapt11 & $92 \cdot 17$ & $2 \cdot 2$ & $1 \cdot 5$ & $0 \cdot 3$ & $0 \cdot 25$ & $0 \cdot 3$ & $3 \cdot 75$ & 97 \\
\hline adapt12 & 93.01 & $1 \cdot 5$ & 0 & 0.557 & $0 \cdot 75$ & $0 \cdot 8$ & $3 \cdot 75$ & 85 \\
\hline adapt22 & 92.94 & 1.5 & $1 \cdot 5$ & 0.554 & $0 \cdot 3$ & $0 \cdot 3$ & 0.75 & 85 \\
\hline adapt25 & $92 \cdot 31$ & 3 & 0.634 & 0.9 & $0 \cdot 25$ & 0.3 & 3 & 85 \\
\hline \multicolumn{9}{|c|}{ Square error (known-adapted)^2 } \\
\hline adapt11 & $1 \cdot 5$ & 0.6 & 0.0 & 0.0 & $0 \cdot 0$ & 0.0 & $0 \cdot 0$ & 169 \\
\hline adapt12 & $4 \cdot 7$ & 0.0 & $2 \cdot 3$ & $0 \cdot 1$ & $0 \cdot 0$ & 0.0 & $0 \cdot 0$ & 0.0 \\
\hline adapt22 & $1 \cdot 6$ & 0.0 & $2 \cdot 3$ & $0 \cdot 1$ & $0 \cdot 2$ & 0.0 & $5 \cdot 1$ & 0.0 \\
\hline adapt25 & $2 \cdot 6$ & 0.0 & 0.7 & $0 \cdot 4$ & 0.3 & 0.3 & 0.0 & 0.0 \\
\hline
\end{tabular}

*The ingredients correspond to: 1-polyether polyol 1; 2polyether polyol 2; 3-amine cross-linker; 4-amine catalyst 1; 5-amine catalyst 2; 6-silicone; 7-water; 8-index. small change (proportional to the PU batch size) in any ingredient for a given formulation is likely to have strong effects on the properties of the foams. Therefore, it is difficult to find a suitable data set to train an ANN. A network likely to learn any mapping between formulation ingredients and mechanical properties would require a vast collection of data covering small variations in the ingredient's quantities. The size of the training data set is also likely to increase as more ingredients are used.

From the PU chemical point of view, certain ingredients have a major impact on the final PU properties. For instance, the catalyst used, and the amount of hydroxyl groups (i.e. coming from water, humidity, polyols, or contaminants) have the highest impact on the stoichiometry of the reaction and hence on the final properties of a foam.

The data sets used for training the ANNs in the present paper, came from a fractional statistical experimental design. In this design of experiments, eight formulation variables were changed simultaneously between the levels suggested by the PU expert to cover the whole gamut of property values. However, it has become apparent that the choice in the change of levels was not refined enough to allow mapping the property formulation spaces continuously evidenced by the wide scatter in properties that resulted. This suggests that for such a large number of formulation variables, more formulations need to be reformulated using smaller changes of the expert's suggested levels to be able to map the space continuously. In addition, changes in the quantity of reactants depend heavily on the type of reactant used, i.e. a change of $5 \mathrm{~g}$ in the amount of catalyst used has a greater effect on the foam final properties than the same change in the amount of the main polyol (for example in a $1 \mathrm{~kg}$ flexible PU foam formulation batch size). Additionally, some ingredients react and produce reactive byproducts, which can, at the same time, lead to unexpected changes in the foam's final properties. As it is, for example, the case of the addition of water and the production of reactive amine that leads to a subsequent production of urea.

\section{Conclusions}

The present paper has studied the use the CBR methodology and ANN approach to support the problems arising in the formulation of PUs. Specifically the hybrid CBR-ANN system allows overcoming the lack of understanding of quantitative models to predict PU formulations given a desired set of mechanical properties in PUs. This has been achieved by structuring and representating expert's PU formulation experiences (i.e. successful and unsuccessful formulation recipes) under a CBR framework for problem solving. Given a problem description or user query, the system was able to retrieve similar experiences with the ultimate objective of predicting what formulation conditions (ingredients and their quantities) allowed the resulting product to meet the desired performance criteria. The CBR-ANN system has shown to have the potential of reducing the time and hence the cost of the product development process while at the same time enabling simulation studies. It also serves as an 'institutional memory' that supports problem solving. 
At its present state of development the described CBR-NN system relies on a relatively limited number of formulation cases. However, it can be readily extended to include new cases as they become available and it is expected that such extension will enhance the usefulness of the presented CBR system. Additionally, the amount of information used to describe a PU formulation case can be increased. For example, the formulation data and hence the description of cases could be complemented with processing information (e.g. mould temperature, mixing time) and chemical data (e.g. \%NCO in the formulation, molecular weight of ingredients) to enhance the usefulness of the system. By enriching the cases with this information, retrieval of cases tailored from different views could be performed and evaluated. In addition, it is expected that if the number of formulations is larger, which can be expected in a research and development department in an industrial setting, improvements in the ANN approach can be made. For example, the results from the ANN training suggested that the main drawback in developing a predictive tool (i.e. able to generalise) that could map mechanical properties to formulation ingredients was the scarcity of data. The cross-validation approach failed to found any network that could learn from the training data presented to the network. Therefore, not only a large data set but careful selection of the training sets is required.

\section{Acknowledgements}

The authors wish to express their gratitude to the industrial collaborators BASF, UK. This work was supported financially by the Engineering and Physical Sciences Research Council (EPSRC).

\section{References}

1. 'Audi A2 goes natural to cut weight', Modern Plast., 2000, 77, (12), 16.

2. BAYER, Baypreg ${ }^{\circledR} \mathrm{F}$ - Polyurethane plus natural materials in cars, 2000, Bayer AG.

3. L. Huber: 'Hot melt polyurethane reactive adhesives targeted for specific applications', available at: www.adhesivesmag.com/asi/cda/ articleinformation/features/bnp_features_item/0,2101,19522,00.html (accessed June 2003).

4. A. M. Howard: Proc. ASE '85, 1-16; 1985, London, Kensington.

5. T. Thomson: 'Polyurethanes as specialty chemicals: principles and applications'; 2004, Boca Raton, FL, CRC Press.

6. N. M. K. Lambda, K. A. Woodhouse and S. L. Cooper: 'Polyurethanes in biomedical applications'; 1998, Boca Raton, FL, CRC Press.

7. P. A. Sykes: 'Structure-property relationships of chain-extended thermoplastic polyurethane elastomers', PhD thesis, Loughborough University, 1999.

8. D. V. Dounis and G. L. Wilkes: Polym. Preprints, 1997, 38, (11), 2819-2828.

9. B. D. Kaushiva, S. R. McCartneya, G. R. Rossmyb and G. L. Wilkes: Polym. Preprints, 2000, 41, 285-310.

10. S. R. Lunney and J. M. Sutej: Proc. 32nd Annu. Conf. on 'Polyurethane technical/marketing', San Francisco, CA, USA, October 1989, SPI, 558.

11. B. Lawrey, N. Barksby, H. Hinney and J. Lear: Proc. UTECH 2000, The Hague, The Netherlands, March 2000, European Isocyanate Producers Association, Paper 10.

12. G. Bellinger: 'Knowledge management - emerging perspectives', available at: www.systems-thinking.org $/ \mathrm{kmgmt} / \mathrm{kmgmt} . \mathrm{htm}$ (accessed January 2004).
13. M. L. Farinacci, M. S. Fox, I. Hulthage and M. D. Rychener: 'The development of ALADIN (aluminum alloy design inventor), an expert system for aluminum alloy design', Interim Report, 1986.

14. I. Hulthage, M. S. Fox and M. D. Rychener and M. L. Farinacci: IEEE Expert, 1990, 5, (4), 56-73.

15. Y. Iwata and N. Obama: Proc. 3rd Conf. on 'Innovative applications of artificial intelligence', Anaheim, CA, USA, July 1991, AAAI Press, 177-191.

16. M. S. Suh, W. C. Jhee, Y. K. Ko and A. Lee: Expert Systems Appl., 1998, 15, (2), 181-190.

17. S. Craw, N. Wiratunga and R. Rowe: Lect. Notes Comput. Sci., 1998, 1488, 358

18. R. Boswell, S. Craw and R. Rowe: Proc. 12th European Conf. on 'Artificial intelligence', Budapest, Hungary, August 1996 Hungarian AI Society, 74-79.

19. S. Bandini and S. Manzoni: 'Knowledge management applications at Pirelli Tires: from P-Race to P-truck projects', available at: www.knowledgeboard.com/cgi-bin/item.cgi?ap=1\&id=111988

20. M. Malek, M. Malek, M.-P. Toitgans, J.-L. Wybo and M. Vincent: Proc. EWCBR98, 402-413; 1998, Berlin, Springer.

21. C. K. Riesbeck and R. Schank: 'Inside case-based reasoning'; 1989 , Erlbaum, Northvale, NJ, Lea.

22. S. Slade: 'Case-based reasoning: a research paradigm', AI Magaz., 1991, 42-55, available at: www.aaai.org/Library/Magazine/Vol12/ 12-01/vol12-01.html

23. R. Heath: Personal communication, Loughborough University, Loughborough, UK, 2005.

24. C. K. Riesbeck and C. K. Riesbeck: 'Introduction to AI programming, lecture notes: CBR', available at: www.cs.northwestern.edu/ riesbeck/ (accessed May 2004).

25. G. E. P. Box, W. Hunter and J. Hunter: 'Statistics for experimenters'; 1978, New York, John Wiley \& Sons.

26. R. Herrington and K. Hock (eds.): 'Flexible polyurethane foams', 1st edn; 1991, Midland, MI, Dow Chemical.

27. 'Flexible cellular polymeric materials - determination of hardness (indentation technique)', BS EN ISO 2439:2001, British Standards Institution, UK

28. 'Flexible cellular polymeric materials - determination of tensile strength and elongation at break', BS EN ISO 1798:2000, British Standards Institution, UK.

29. 'Flexible cellular polymeric materials - determination of compression set', BS EN ISO 1856:2001, British Standards Institution, UK.

30. J. L. Kolodner: 'Case-based reasoning'; 1993, San Mateo, CA Morgan Kaufmann Publishers.

31. S. Bandini and S. Manzoni: Proc. 4th Int. Conf. on 'Case-based reasoning', Vancouver, Canada, July-August 2001, SpringerVerlag GmbH, 634-647.

32. N. Wiratunga, S. Craw and S. Massie: Lect. Notes Comput. Sci., 2003, 2689, 637-651.

33. A. F. Smeaton and C. J. V. Rijsbergen: Proc. Annu. ACM Conf. on 'Research and development in information retrieval', Oakland, CA, USA, May-June 1981, ACM, 83-87.

34. W. Wilke and R. Bergmann: Proc. 11th Int. Conf. on 'Industrial and engineering applications of artificial intelligence and expert systems', Benicassim, Castellon, Spain, June 1998, Springer-Verlag, 497-506.

35. K. Börner: Artific. Intellig. Rev., 2001, 16, (2), 87-118.

36. S. Craw, J. Jarmulak and R. Rowe: Proc. 4th Int. Conf. on 'Casebased reasoning', Vancouver, Canada, July-August 2001, SpringerVerlag, 131-145.

37. K. Hanney and M. Keane: Proc. 2nd Int. Conf. on 'Case-based reasoning', 359-381; 1997, Providence, RI, Springer.

38. J. Jarmulak, S. Craw and R. Rowe: Proc. 17th Int. Joint Conf. on 'Artificial intelligence' Seattle, WA, USA, August 2001, Morgan Kaufmann, 1011-1016.

39. T. Masters: 'Practical neural network recipes in $\mathrm{C}++$ '; 1993, Boston, MA; London, Academic Press.

40. R. Hecht-Nielsen: 'Neurocomputing'; 1990, Wokingham, AddisonWesley.

41. M. T. Hagan, H. B. Demuth and M. Beale: 'Neural network design'; 1996, Boston, MA, PWS Publishing Company.

42. C. Bishop: 'Neural networks for pattern recognition'; 1995 , Oxford, Clarendon Press. 